Check for updates

Cite this: Phys. Chem. Chem. Phys., 2018, 20, 14145

Received 20th February 2018 Accepted 24th March 2018

DOI: $10.1039 / c 8 c p 01156 g$

rsc.li/pccp

\section{Imperfect mixing as a dominant factor leading to stochastic behavior: a new system exhibiting crazy clock behavior}

\begin{abstract}
László Valkai and Attila K. Horváth (D) *
It is clearly demonstrated that the arsenous acid-periodate reaction displays crazy-clock behavior when a statistically meaningful number of kinetic runs are performed under "exactly the same" conditions. Both extensive experimental and numerical simulation results gave convincing evidence that the stochastic feature of the title reaction originates from the imperfection of the mixing process, and neither local random fluctuation nor initial inhomogeneity alone is capable of explaining adequately the observed phenomena. Imperfect mixing is manifested-in practice-in the unintentional and inherent formation of dead volumes where the concentration of the reactants may even significantly differ from the ones measured in the case of a completely uniform concentration distribution, and the system may spend enough time there under imperfectly mixed conditions to complete the nonlinear chemical process. Furthermore, it is also shown that a more efficient mixing, i.e. a smaller dead volume size and shorter residence time being spent in the dead volume, does not necessarily mean Landolt times are smaller than the one measured under completely homogeneous conditions. Evidently, the "initial" concentration of the reagents in the dead volume-and of course in the rest of the solution-greatly influences the Landolt time to be measured in the case of an individual kinetic run and may therefore show either positive or negative deviation from the Landolt time for the completely homogeneous state. As a result, less efficient mixing may either accelerate or decelerate the rate of a nonlinear autocatalytic reaction at a macroscopic volume level.
\end{abstract}

\section{Introduction}

Experimental and theoretical investigations of the origin of stochastic features in real chemical or biological systems have received considerable general interest since the discovery of the first two Landolt-type redox systems exhibiting serious irreproducibility in measuring the time necessary for the sudden appearance of a product. When parallel experiments were performed under "exactly the same experimental conditions" in the chlorite-thiosulfate ${ }^{1}$ and iodide-chlorite ${ }^{2}$ reactions, the products of chlorine dioxide and iodine, respectively, appeared after a fairly long but strongly irreproducible time lag, making the reactions later be classified as crazy-clock reactions. ${ }^{3}$ The phenomenon became the subject of great interest when Soai et al. reported the discovery of asymmetric amplification in an autocatalytic reaction in $1995,{ }^{4}$ followed by their subsequent study on the stochastic nature of enantiomerically enriched pyrimidyl alkanol obtained from the reaction of pyrimidine-5carbaldehyde and diiodopropylzinc. ${ }^{5}$ Since then, these reactions

Faculty of Sciences, Department of Inorganic Chemistry, University of Pécs, Ifjúság útja 6, Pécs, Hungary. E-mail: horvatha@gamma.ttk.pte.hu have became the prototypes of stochastic chemical systems. The application of stochastic kinetics in biology and chemistry has recently been summarized in a comprehensive book by Érdi and Lente. ${ }^{6}$ The mathematical description is also available and a lot of theories and algorithms are discussed in detail, but their usage is often limited due to the fact that a complete description of the deterministic kinetics of these systems is either not available or their mechanisms are too complicated. Furthermore, Lente has recently shown that in the case of a simple autocatalytic system, the probability distribution of Landolt times can be approximated by an appropriate time shift property of distributions. This approximation works pretty well and is capable of providing the stochastic master equation of the system without any limitation regarding the size of the system. ${ }^{7}$ To visualize the difficulties of describing the quantitative behavior of a stochastic chemical system, so far, still no acceptable kinetic model has been suggested for characterizing the most important nonlinear dynamical features of the chlorite-thiosulfate and the Soai reactions, despite the fact that tentative models containing many elementary and non-elementary steps exist for both systems to explain some of their exotic kinetic characteristics. ${ }^{8,9}$ Furthermore, a comprehensive kinetic model of the iodide-chlorite system was reported in 
1996, but this model is quite complicated and involves many important oxyhalogen intermediates. ${ }^{10}$ It is therefore expected that a simpler real chemical system may facilitate the study of the theoretical background of the emergence of stochastic behavior. This expectation, along with the fact that the arsenous acid-iodate reaction exhibits crazy-clock behavior (CCB) as reported recently by our research group, ${ }^{11}$ has led us to elucidate the comprehensive kinetic model of the arsenous acid-iodate system, ${ }^{12,13}$ including the well-known Dushman ${ }^{14}$ and Roebuck ${ }^{15}$ reactions. Very recently, Pagnacco et al. reported $^{16}$ that the state I (low iodide and iodine concentration) to state II (high iodide and iodine concentration) transition of the Briggs-Rauscher reaction ${ }^{17}$ after leaving the strongly reproducible oscillatory state was found to be irreproducible as well. Moreover, an efficient mixing does not only prolong the time lag necessary for the state I to state II transition, it simply does not allow it to happen. This study further extended the number of systems exhibiting CCB, though the complete kinetic description of this complex system is still in dispute. ${ }^{18-20} \mathrm{~A}$ principle for explaining the origin of stochasticity was proposed by Nagypál and Epstein in their preliminary work. ${ }^{1,2}$ As discussed in these papers, in an autocatalytic redox system, random and local fluctuation usually occurs at a later phase of the given reaction. In these particular places (the so-called ignition volumes), the autocatalytic process is ignited and the concentration of key species increases rapidly there. Upon diffusion of the autocatalyst to the closest unreacted zones, the autocatalytic process is again ignited in the close vicinity of the ignition volume to complete the process in an extended volume to increase the concentration of the autocatalyst further. The chemical wave generated this way then finally spreads through the entire bulk of the solution. This idea was supported by the fact that the increase of the overall volume significantly shortens the Landolt time, because the probability that random fluctuations produce more and more ignition places indeed increases. In our previous report when using the arsenous acid-iodate reactions to study the origin of $\mathrm{CCB},{ }^{11}$ however, it was convincingly proven by systematically varying the stirring rate, the way of mixing, and the geometry of the reactor, that the fate of individual samples in this reaction is determined at the beginning stage-upon mixing the reactants-when the system is per se inhomogeneous. This study made it clear that even if random fluctuation at the later stage of an autocatalytic reaction cannot be entirely ruled out as a source of stochasticity, inherent inhomogeneity must play a crucial role in causing the serious irreproducibility of the individual samples performed "under exactly the same experimental conditions". A straightforward question may immediately be raised, whether the inherent inhomogeneity in the case of an autocatalytic reaction is the only factor that leads directly to stochastic behavior, or whether there must be other-so far hidden-components. Finding the early results of Abel and Fürth on the periodate-arsenous acid reaction ${ }^{21,22}$ gave us an idea that it is possible to produce iodate in situ to study whether we can or cannot eliminate CCB via introducing iodate essentially homogeneously by the relatively slow periodate-arsenous acid reaction. The most important experimental findings and the conclusions to be drawn are reported below.

\section{Experimental section}

\subsection{Materials}

All of the materials were of the highest purity commercially available and used without further purification. Some of the chemicals, such as sodium arsenite, potassium periodate, methyl red, thymolphthalein, potassium carbonate and potassium hydrogencarbonate, were purchased from Reanal, while others, like sodium dihydrogen phosphate, hydrochloric acid and sodium perchlorate, were obtained from Sigma-Aldrich. Concentrated phosphoric acid and solid sodium hydroxide were bought from Merck and VWR, respectively. The concentrations of the acid solutions were determined using standard titration processes, while the concentration of the periodate solution was checked spectrophotometrically. ${ }^{23}$ The concentration of the arsenite stock solution was regularly determined using a routine procedure described elsewhere. ${ }^{13}$

All of the stock solutions were prepared from twice ionexchanged and twice distilled water. The ion-exchanged water was first distilled from potassium permanganate, then atmospherically to eliminate all carbon containing, redox active impurities from the system.

\subsection{Instruments}

An Analytik Jena SPECORD S600 diode array spectrophotometer was used for measuring the UV-Vis spectra. Calibrated Eppendorf Reference automatic pipettes were used for precise volume measurements.

\subsection{Methods}

A large volume of each stock solution was prepared to carry out each and every measurement "under exactly the same" initial conditions. This way, one can easily avoid changes in the quantities of trace impurities that are unintentionally present in the system. The total periodate and dihydrogen-arsenite concentrations were set to $6.50 \mathrm{mM}$ and $6.35 \mathrm{mM}$, respectively. The ionic strength and the $\mathrm{pH}$ were adjusted using a phosphate buffer to $0.20 \mathrm{M}$ and 1.50 , respectively.

The reaction was carried out in a cylindrical vessel and its diameter and the length of the stirrer bar used were systematically varied, as shown in Tables 1 and 2, respectively. The height of the solution was set to the same level in all of the experiments. For every different setup, 50 individual experiments were carried out to obtain a cumulative distribution function. The Landolt time was detected by the naked eye in almost all of the experimental series, as iodine has a characteristic visible absorption, except in one series containing 13 measurements, where the kinetic runs were followed spectrophotometrically.

Table 1 Physical parameters of the reactors

\begin{tabular}{llc}
\hline Name & Inner diameter $/ \mathrm{mm}$ & Nominal volume $/ \mathrm{mL}$ \\
\hline Tiny cylinder & 10.4 & 3 \\
Small cylinder & 11.9 & 5 \\
Big cylinder & 17.4 & 10 \\
Vial & 23.9 & 20 \\
Beaker & 26.9 & 25
\end{tabular}


Table 2 Physical parameters of the stirrer bars

\begin{tabular}{lll}
\hline Length $/ \mathrm{mm}$ & Diameter $/ \mathrm{mm}$ & Volume $/ \mathrm{mL}$ \\
\hline 9.9 & 5.8 & 0.273 \\
13.0 & 8.0 & 0.508 \\
20.0 & 5.0 & 0.458
\end{tabular}

An additional three sets of experiments were done in a round-bottomed flask, equipped with a cross-shaped magnetic stirrer that had a diameter of $1 \mathrm{~cm}$. The stirring rate was controlled at $500 \mathrm{rpm}$ in every experiment.

To avoid any disturbance effect, the experiments were tried to be carried out using exactly the same procedure. The initiating agent was always the arsenite solution in each run and altogether 663 individual kinetic runs were collected by one experimentalist.

For the simulations and evaluation of the results, the program package ChemMech ${ }^{24}$ was used.

\section{Results and discussion}

A sequence of reactions describing the kinetics of the periodate-arsenous acid reaction starts with the production of iodate via a relatively slow reaction, represented by eqn (1). The rate coefficient of this step was found to be $5.5 \mathrm{M}^{-1} \mathrm{~s}^{-1}$ by Abel and Fürth, ${ }^{21,22}$ indicating that iodate may be relatively slowly produced during the course of the reaction. This makes it possible to avoid its instantaneous but necessarily inhomogeneous addition compared to in our previous study in the case of the arsenous acid-iodate reaction. ${ }^{11}$ For the sake of completeness, it should be emphasized that periodate exists in its octahedral form in an overwhelming excess over the tetrahedral form in the aqueous solution, ${ }^{23}$ therefore we consider the former form to be the kinetically active species.

$$
\mathrm{H}_{4} \mathrm{IO}_{6}{ }^{-}+\mathrm{H}_{3} \mathrm{AsO}_{3} \rightarrow \mathrm{IO}_{3}{ }^{-}+\mathrm{H}_{3} \mathrm{AsO}_{4}+2 \mathrm{H}_{2} \mathrm{O}
$$

The iodate ion formed in this process is then reduced further by arsenous acid to eventually produce an iodide ion

$$
3 \mathrm{H}_{3} \mathrm{AsO}_{3}+\mathrm{IO}_{3}^{-} \rightarrow 3 \mathrm{H}_{2} \mathrm{AsO}_{4}^{-}+\mathrm{I}^{-}+3 \mathrm{H}^{+}
$$

that ignites the Dushman reaction ${ }^{14}$ to yield iodine

$$
\mathrm{IO}_{3}^{-}+5 \mathrm{I}^{-}+6 \mathrm{H}^{+} \rightarrow 3 \mathrm{I}_{2}+3 \mathrm{H}_{2} \mathrm{O} .
$$

Finally, iodine rapidly oxidizes arsenous acid to reform the iodide ion in a relatively rapid process:

$$
\mathrm{H}_{3} \mathrm{AsO}_{3}+\mathrm{I}_{2}+\mathrm{H}_{2} \mathrm{O} \rightarrow \mathrm{H}_{2} \mathrm{AsO}_{4}{ }^{-}+2 \mathrm{I}^{-}+3 \mathrm{H}^{+} .
$$

It is therefore straightforwardly expected that under acidic conditions where the Dushman reaction is rapid enough, the system behaves as an iodine-clock reaction because iodine suddenly appears after a fairly long period of time. Our preliminary experiments, however, unambiguously indicated that this time lag varies in an apparently random manner in parallel runs. To demonstrate the CCB in this system, we carried out some experiments in a continuously stirred sealed cuvette and recorded the visible spectra of the solution. The results of four

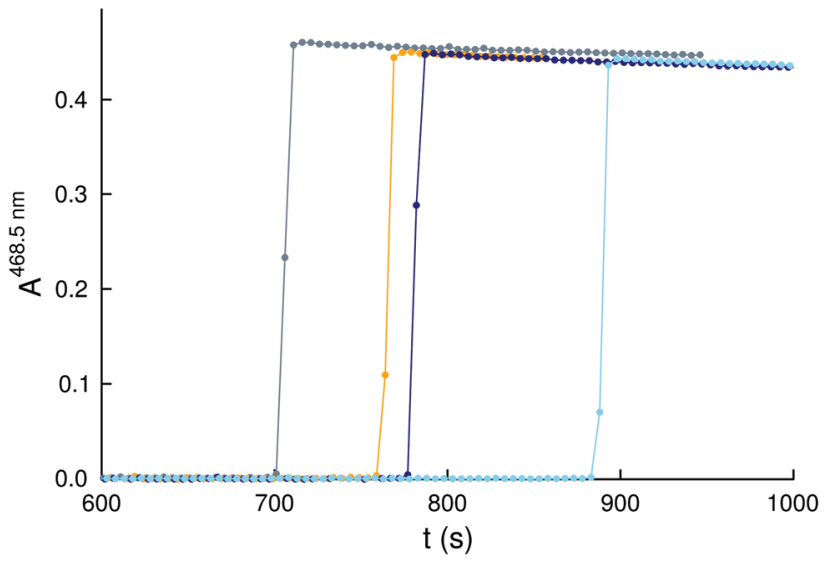

Fig. 1 Repetitions of the kinetic runs in the periodate-arsenic acid system. $T_{1}=6.50 \mathrm{mM}, T_{\mathrm{As}}=6.35 \mathrm{mM}, \mathrm{pH}=1.40, I=0.20 \mathrm{M}$, and the stirring rate is $500 \mathrm{rpm}$.

different kinetic curves that are shown in Fig. 1 clearly support the fact that the length of the induction period for an individual kinetic run varies in a random manner between 700 and $900 \mathrm{~s}$. For the sake of completeness, it should be emphasized that the iodine concentration slowly decreases at the final stage of the reaction. Possibly, slow direct or indirect (via $\mathrm{HOI}$ and/or $\mathrm{HIO}_{2}$ ) oxidation of iodine by the excess of periodate ions occurs in the final stage. ${ }^{25}$ This side reaction, however, does not play any significant role in the crazy-clock behavior, therefore it is neglected in the subsequent discussion. The clear observation of CCB immediately raises the following questions. Does this feature of the arsenous acid-periodate reaction uniquely belong to the characteristics of the arsenous acid-iodate system, or is this reaction alone capable of exhibiting CCB? Does the appearance of CCB mean that random fluctuation at a later phase of the reaction determines the fate of an individual sample in the case of the arsenous acid-periodate system, or is it simply the consequence of imperfect mixing? To answer these questions, we performed systematic studies with the variation of several physical parameters of the mixing process by carrying out a statistically meaningful number of experiments (usually fifty or even more) under the same experimental conditions. From the Landolt times determined experimentally, a cumulative probability distribution was constructed as was demonstrated previously. ${ }^{111}$ If we assume a normal distribution, we may easily fit the following function to the measured points:

$$
\Phi\left(\frac{t_{\mathrm{L}}-\mu}{\sigma}\right)=50 \%\left(1+\operatorname{erf}\left(\frac{t_{\mathrm{L}}-\mu}{\sigma \sqrt{2}}\right)\right)
$$

where $\Phi$ is the cumulative distribution function, $t_{\mathrm{L}}$ is the Landolt time, $\mu$ is the mean of the distribution, $\sigma$ is the standard deviation and $\operatorname{erf}()$ denotes the error function. The results of these fits are demonstrated in a number of different experimental setups (see Fig. 2-5), justifying a sound agreement between the measured and calculated data.

In the first set of experiments the size of the reactor was varied, while the same stirrer bar was used (the smallest one). 
Consequently, our experimental setup means that the overall volume also increases with an increase of the diameter of the reactor. As Fig. 2 shows, the most probable Landolt time is longer as the reactor size, hence the volume, increases. Furthermore, the quality of the fit is more perfect in the middle region (the corresponding errors are as follows: 11\%, 18\%, 11\%, 7\%, and $9 \%)$.

This observation completely matches our previous result reported in the arsenous acid-iodate system, but differs totally from the main outcome of Nagypál and Epstein, where it was demonstrated that an increase of the volume significantly shortens the Landolt times. Careful inspection of Fig. 2 reveals that the Landolt time belonging to the $50 \%$ probability is slightly shortened when the diameter of the reactor increases from 2.4 to $2.7 \mathrm{~cm}$, which correspond to overall volumes of 4.67 and $5.4 \mathrm{~mL}$. To ensure that this changing trend is not an artifact, we have repeated our experiments at larger overall volumes as well. This result is shown in Fig. 3.

First, it is clearly shown that no significant effect is observed in the most probable Landolt time $(\mu)$ when the number of places of initiation is varied, but at the same time $\sigma$ looks to be larger when only a single initiation point is used. Although it can easily be rationalized (see: later, the effect of the concentration distribution of the reactants in the dead volume), a direct comparison of $\sigma$ values is difficult to make due to the fact that the numbers of the individual experiments performed significantly differs from each other. Second, it is not surprising that when the overall volume was increased from 4.8 to $7.2 \mathrm{~mL}$, the Landolt time belonging to the $50 \%$ probability became significantly shorter. It is exactly the same trend observed by Nagypál and Epstein when varying the overall volume from 4.0 to $10.0 \mathrm{~mL}$ in the chlorite-thiosulfate reaction! ${ }^{1}$ In other words, it means that in the case of the arsenous acid-periodate reaction, the Landolt time belonging to the 50\% probability shows a maximum as a function of volume. For the sake of completeness, it should also be mentioned that in the case of Fig. 2 a small stirrer bar is used, while in the case of Fig. 3 a cross-shaped stirrer is used to mix the solutions.

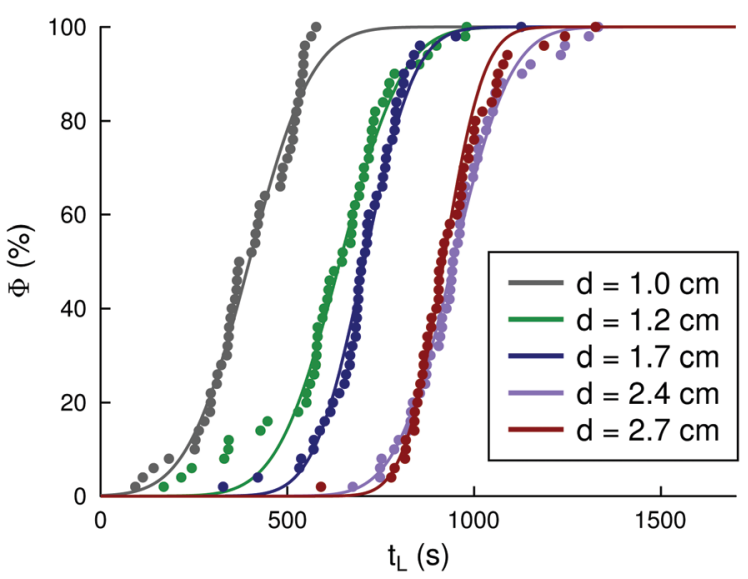

Fig. 2 Varying the diameter of the cylindrical reactor, using the smallest stirrer bar.

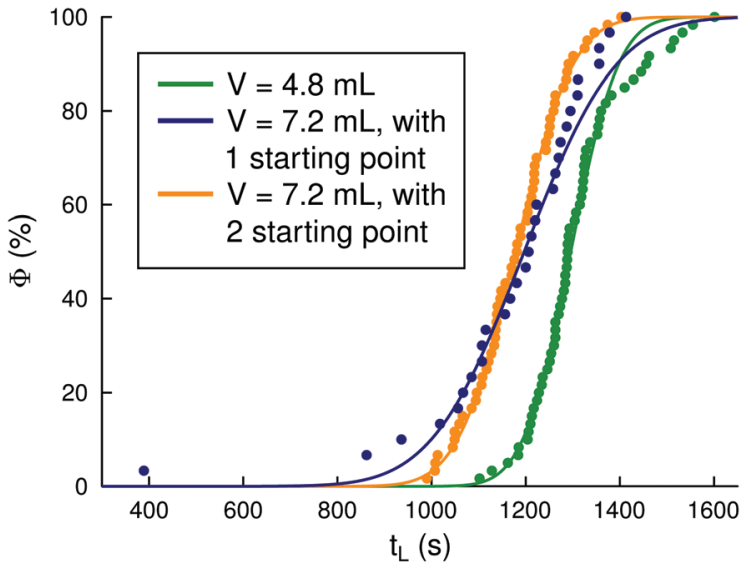

Fig. 3 Cumulative distribution curves obtained in the case of a $25 \mathrm{~mL}$ round bottomed flask equipped with a cross-shaped stirrer bar with a diameter of $1 \mathrm{~cm}$ at two different overall volumes. The experiments at a total volume of $7.2 \mathrm{~mL}$ were carried out by adding the initializing agent from one (blue curve) or two (orange curve) pipettes.

As a result, the most probable Landolt time significantly increases when the latter stirrer $\left(\mu=1296 \mathrm{~s}, V_{\text {overall }}=4.8 \mathrm{~mL}\right)$ is used instead of the former $\left(\mu=944 \mathrm{~s}, V_{\text {overall }}=4.67 \mathrm{~mL}\right)$ at almost the same overall volume. This observation appears to suggest that not only does the initial inhomogeneity play a crucial role in determining the Landolt time-as proposed in our previous work ${ }^{11}$ - but the stirring pattern generated by the stirrer bar and the geometry of the reactor must also affect the fate of an individual sample via inherently providing dead volumes with different sizes and different residence times for the imperfectly mixed solution staying there. This hypothesis may further be supported by varying the place of initiation. Fig. 4 displays the result in the case of a statistically meaningful number of samples at two different setups: when the place of initiation was right in the center of the vortex or it was placed approximately halfway between the wall of the reactor and the center of the vortex. As can be clearly seen, significantly longer Landolt times can be measured when the initiation is performed right in the middle of the vortex. This result is in complete agreement with our previous findings in the case of the iodate-arsenous acid reaction. ${ }^{11}$ To confirm our hypothesis mentioned previously, the movement of the stirrer bar was varied in the next set of experiments at a constant mixing rate. In one of the series, the rotation axis of the stirrer bar was completely fixed, while in the other case, the stirrer bar was allowed not only to rotate around a fixed axis but it was also able to wander. The result is shown in Fig. 5. As this figure clearly shows, different stirring control has a relatively huge effect on the most probable Landolt time. When the stirrer bar was allowed to wander, i.e., its rotating position was not fixed at a standard axis, $\mu$ (798 s) became significantly shorter compared to the other case $(\mu=944 \mathrm{~s})$. This observation also proves that the stirring pattern indeed has to have a major contribution to the Landolt time. As a result, one may easily expect that changing the size of the stirrer should also affect the measured Landolt time because evidently the stirring pattern must be different when the 


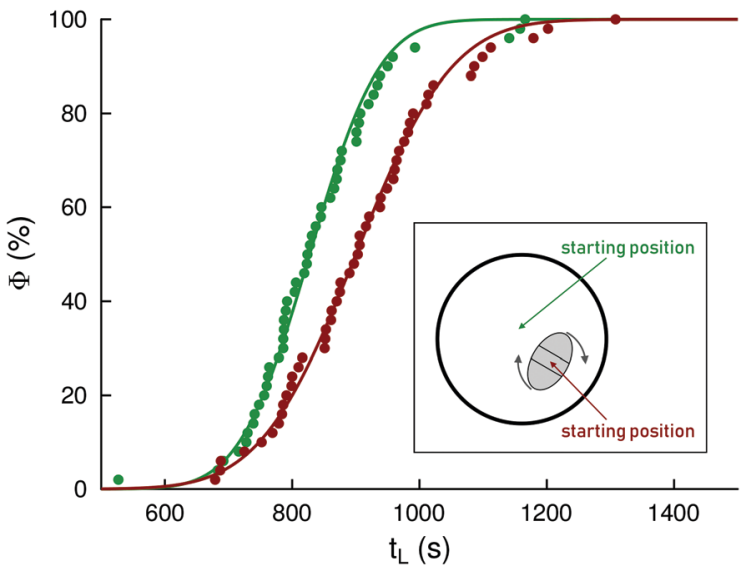

Fig. 4 Varying the starting position, while the rest of the physical parameters are the same. The middle-sized stirrer bar and the largest cylindrical reactor are used. The green color represents the case when the starting position was set halfway between the vortex and the wall of the reactor, while the red color corresponds to the case when the initiation was right in the center of the vortex.

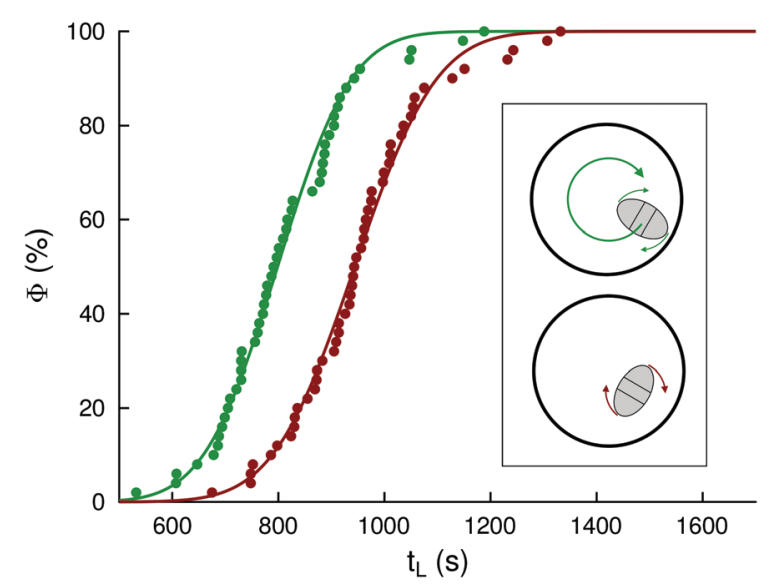

Fig. 5 Variation of the stirring pattern, with the use of the same reactor and stirrer bar. The colors representing the stirring pattern belong to the same colored curves.

same overall volume is stirred at the same stirring rate in the same reactor by using stirrer bars with different sizes. Therefore the next series of experiments was carried out by varying the size of the stirrer bar. Consequently, our experimental setup means that the total volume was slightly changed as well. The results are represented in Fig. 6.

As can be seen in contrast to our expectation, however, we found no effect of changing the size of the stirrer bar on the most probable Landolt time ( $\mu$ was found to be 918, 900 and $905 \mathrm{~s}$, when the length was varied to $9.9,13.0$ and $20.0 \mathrm{~mm}$, respectively). One possible explanation of this experimental result is that changing the size of the stirrer bar may not have a significant effect as expected. But at the same time we would like to stress that increasing the size of the stirrer bar means a larger volume according to our experimental setup and thus the increasing volume indeed has a notable effect. Following this argument, if we accept that both the initial inhomogeneity and

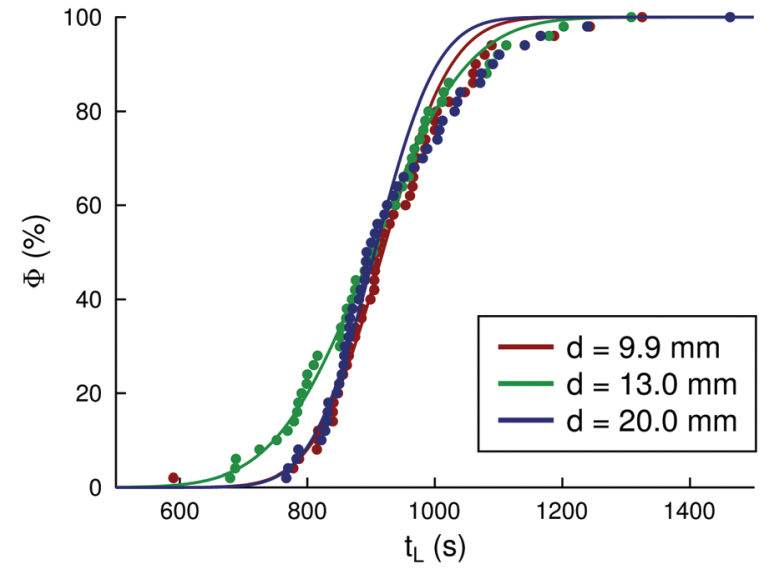

Fig. 6 Changing the stirrer bar, while the reactor is the same (the largest one).

the stirring pattern have a strong influence on the apparent insensitivity of Landolt times when the size of the stirrer bar is varied, then it may only be explained if the effects of these forces compensate for each other.

To rationalize all of the experimental results mentioned above, the effect of inhomogeneity being introduced artificially in a Landolt-type deterministic kinetic model is studied using extensive numerical simulations. The following deterministic kinetic model is used throughout the complete numerical study:

$$
\begin{array}{cc}
\mathrm{P}+\mathrm{Q} \rightarrow \mathrm{R} & v_{1}=k_{1}[\mathrm{P}][\mathrm{Q}] \\
\mathrm{R}+\mathrm{Q} \rightarrow \mathrm{C} & v_{2}=k_{2}[\mathrm{R}]^{2}[\mathrm{Q}] \\
\mathrm{P}+\mathrm{C} \rightarrow 2 \mathrm{R} & v_{3}=k_{3}[\mathrm{P}][\mathrm{C}]
\end{array}
$$

where P, Q, C and R may represent, for instance, arsenous acid, iodate or periodate, iodine and iodide, respectively. It is easy to see that if the $[\mathrm{Q}]_{0}>[\mathrm{P}]_{0}$ inequality is fulfilled, then species $\mathrm{C}$ appears suddenly, when species $\mathrm{P}$ is completely consumed and $k_{3}$ is large enough. The first simulation was performed at constant initial concentrations, denoted $[\mathrm{P}]_{0}$ and $[\mathrm{Q}]_{0}$, supposing a completely uniform concentration distribution in the entire volume $\left(V_{\text {total }}\right)$ of the solution. The time lag (the so-called Landolt time) necessary for the sudden appearance of $\mathrm{C}$ is denoted $t_{\mathrm{L}}$. Following the concept of Horváth and Nagypál, ${ }^{3}$ the analytical solution of the differential equation governing this system may easily be derived to calculate $t_{\mathrm{L}}$ :

$$
\begin{aligned}
t_{\mathrm{L}}= & \frac{\ln \left(\frac{[\mathrm{Q}]_{0}}{[\mathrm{Q}]_{0}-[\mathrm{P}]_{0}} \sqrt{\frac{k_{2}[\mathrm{P}]_{0}}{k_{1}}}\right)}{k_{1}\left([\mathrm{P}]_{0}-[\mathrm{Q}]_{0}\right)+k_{2}[\mathrm{Q}]_{0}^{2}} \\
& +\frac{2 k_{2}[\mathrm{Q}]_{0}-k_{1}}{\sqrt{4 k_{1} k_{2}[\mathrm{P}]_{0}-k_{1}^{2}}} \arctan \frac{4 k_{2}[\mathrm{P}]_{0}-k_{1}}{k_{1}\left([\mathrm{P}]_{0}-[\mathrm{Q}]_{0}\right)+k_{2}[\mathrm{Q}]_{0}^{2}}
\end{aligned}
$$

where $\ln ()$ and $\arctan ()$ are the natural logarithm and the arcus tangent functions. The mathematical background of the derivation of eqn (5) may be found elsewhere. ${ }^{26}$ We then introduced artificial 
deviations from both $[\mathrm{P}]_{0}$ and $[\mathrm{Q}]_{0}$ values for a certain period of time $\left(t_{\mathrm{i}}\right)$ in a small portion $\left(V_{\text {dead }}\right)$ of the overall volume. If we consider that the concentration of species $\mathrm{P}$ is $[\mathrm{P}]_{\text {stock }}$ in a $V_{\mathrm{P} \text {,stock }}$ volume present in the reactor and the reaction is started by injecting species $\mathrm{Q}$ in a volume of $V_{\mathrm{Q}, \text { stock }}$ and a concentration of $[\mathrm{Q}]_{\text {stock }}$, then of course the concentrations of species $\mathrm{P}$ and $\mathrm{Q}$ initially in the dead volume may only vary between 0 to $[\mathrm{P}]_{\text {max,dead }}$ and 0 to $[\mathrm{Q}]_{\text {max,dead }}$. The expression $[\mathrm{A}]_{\text {max,dead }}=[\mathrm{A}]_{\text {stock }} V_{\text {total }} / V_{\mathrm{A}, \text { stock }}$ provides the value of the upper limit, where A may correspond to the $\mathrm{P}$ or $\mathrm{Q}$ species. Furthermore, considering that during the mixing process small parcels of solutions containing species $\mathrm{P}$ and $\mathrm{Q}$ are placed next to each other, the values of $[\mathrm{P}]_{0, \text { dead }}$ and $[\mathrm{Q}]_{0, \text { dead }}$ cannot be independent of each other. If the dead volume consists of a volume $V_{1}$ of species $\mathrm{P}$ then $[\mathrm{P}]_{0, \text { dead }}=$ $[\mathrm{P}]_{\text {max,dead }} V_{1} / V_{\text {dead }}$ and $[\mathrm{Q}]_{0 \text {,dead }}=[\mathrm{Q}]_{\text {max,dead }}\left(V_{\text {dead }}-V_{1}\right) / V_{\text {dead }}$ must be fulfilled. To perform the calculations within the dead volume, we considered a uniform concentration distribution of species $\mathrm{P}$ and $\mathrm{Q}$. At the same time in the rest of the reactor, reactant concentrations were calculated from the mass balance. Hence the equation $[\mathrm{A}]_{0, \text { bulk }}=\left([\mathrm{A}]_{0} V_{\text {total }}-[\mathrm{A}]_{0, \text { dead }} V_{\text {dead }}\right) /\left(V_{\text {total }}-V_{\text {dead }}\right)$ gives the initial concentrations of the $\mathrm{P}$ and $\mathrm{Q}$ species outside the dead volume. In this expression, A may correspond to the $\mathrm{P}$ and $\mathrm{Q}$ species as well. This way, we obtained two different parts of the overall volume within which the concentration distribution is uniform, but the exact values may even differ significantly from each other. In these two regions, the concentrations of the $\mathrm{P}, \mathrm{Q}$ and $\mathrm{R}$ species are calculated up to the $t_{\mathrm{i}}$ time point in separate calculations supposing that no transport process occurs between these regions. This time point is systematically increased, meaning that the system may remain inhomogeneous for a longer period of time. Exactly at the $t_{\mathrm{i}}$ time point, we "mixed" these solutions to provide a completely uniform concentration distribution in the entire volume. At this moment, we may consider that the new initial concentrations of $[\mathrm{P}]_{0}=[\mathrm{P}]_{t_{t}},[\mathrm{Q}]_{0}=[\mathrm{Q}]_{t_{\mathrm{i}}}$ and $[\mathrm{R}]_{0}=[\mathrm{R}]_{t_{\mathrm{i}}}$ are valid, thus $t_{\mathrm{h}}$ can be calculated from the following equation:

$$
\begin{gathered}
t_{\mathrm{h}}=\frac{\ln \left(\frac{[\mathrm{Q}]_{0}}{[\mathrm{Q}]_{0}-[\mathrm{P}]_{0}} \sqrt{\frac{k_{2}[\mathrm{P}]_{0}^{2}+k_{2}[\mathrm{R}]_{0}^{2}}{k_{1}[\mathrm{P}]_{0}+k_{2}[\mathrm{R}]_{0}^{2}}}\right)}{k_{1}\left([\mathrm{P}]_{0}-[\mathrm{Q}]_{0}\right)+k_{2}\left([\mathrm{Q}]_{0}^{2}+[\mathrm{R}]_{0}^{2}\right)+2 k_{2}[\mathrm{R}]_{0}[\mathrm{Q}]_{0}} \\
+\frac{2 k_{2}[\mathrm{Q}]_{0}-k_{1}+2 k_{2}[\mathrm{R}]_{0}}{\sqrt{4 k_{1} k_{2}\left([\mathrm{P}]_{0}+[\mathrm{R}]_{0}\right)-k_{1}^{2}}} \arctan \frac{2[\mathrm{P}]_{0} \sqrt{4 k_{1} k_{2}\left([\mathrm{P}]_{0}+[\mathrm{R}]_{0}\right)-k_{1}^{2}}}{2 k_{1}[\mathrm{P}]_{0}+4 k_{2}[\mathrm{R}]_{0}\left([\mathrm{P}]_{0}+[\mathrm{R}]_{0}\right)} \\
k_{1}\left([\mathrm{P}]_{0}-[\mathrm{Q}]_{0}\right)+k_{2}\left([\mathrm{Q}]_{0}^{2}+[\mathrm{R}]_{0}^{2}\right)+2 k_{2}[\mathrm{R}]_{0}[\mathrm{Q}]_{0}
\end{gathered}
$$

Details of the derivation of eqn (6) may be found elsewhere. ${ }^{26}$ The Landolt time $\left(t_{\mathrm{L}}\right)$ is thus calculated as $t_{\mathrm{L}}=t_{\mathrm{i}}+t_{\mathrm{h}}$. During the simulation, $k_{1}=10^{-5} \mathrm{M}^{-1} \mathrm{~s}^{-1}, k_{2}=1.5 \times 10^{6} \mathrm{M}^{-2} \mathrm{~s}^{-1}, k_{3}=$ $10^{6} \mathrm{M}^{-1} \mathrm{~s}^{-1}, P_{0}=7.0 \mathrm{mM}, Q_{0}=10.0 \mathrm{mM}$ and $V_{\text {total }}=3.0 \mathrm{~mL}$ are used. Table 3 summarizes the calculated Landolt times at different $t_{\mathrm{i}}$ values, within which the system stayed in an inhomogeneous state.

As one may easily notice, $t_{\mathrm{L}}$ calculated in this way is larger in the majority of the simulated runs compared to the $t_{\mathrm{L}}$ value belonging to the completely uniform concentration distribution (see the first row of Table 3). However, the trend of $t_{\mathrm{L}}$ as a
Table 3 Calculated Landolt times at different $t_{\mathrm{i}}$ values and at $V_{\text {dead }}=$ $0.3 \mathrm{~mL}$. The initial conditions are as follows: $V_{\mathrm{P}, \text { stock }}=2.0 \mathrm{~mL}, V_{\mathrm{Q}, \text { stock }}=$ $1.0 \mathrm{~mL}$ and $V_{1}=0.285 \mathrm{~mL}$ (upper part of the table), $V_{1}=0.075$ (middle part)

\begin{tabular}{|c|c|c|c|c|}
\hline$t_{\mathrm{i}}(\mathrm{s})$ & $R_{t_{\mathrm{i}} \text {,dead }}(\mu \mathrm{M})$ & $R_{t_{\mathrm{i}} \text {,bulk }}(\mu \mathrm{M})$ & $t_{\mathrm{h}}(\mathrm{s})$ & $t_{\mathrm{L}}(\mathrm{s})$ \\
\hline 0 & 0 & 0 & 542.01 & 542.01 \\
\hline 10 & 0.0014963 & 0.0073017 & 532.41 & 542.41 \\
\hline 30 & 0.0044891 & 0.0219611 & 513.20 & 543.20 \\
\hline 60 & 0.0089804 & 0.0443066 & 484.31 & 544.31 \\
\hline 120 & 0.0179781 & 0.0918572 & 425.93 & 545.93 \\
\hline 180 & 0.0270108 & 0.1469210 & 366.23 & 546.23 \\
\hline 240 & 0.0360963 & 0.2165940 & 304.61 & 544.61 \\
\hline 300 & 0.0452529 & 0.3153470 & 240.61 & 540.61 \\
\hline 360 & 0.0544994 & 0.4804180 & 173.96 & 533.96 \\
\hline 420 & 0.0638554 & 0.8503790 & 104.68 & 524.68 \\
\hline 480 & 0.0734124 & 2.7768700 & 33.17 & 513.17 \\
\hline 10 & 0.0059094 & 0.0063940 & 532.88 & 542.88 \\
\hline 30 & 0.0178039 & 0.0192204 & 516.14 & 544.61 \\
\hline 60 & 0.0361313 & 0.0387043 & 487.19 & 547.19 \\
\hline 120 & 0.0768481 & 0.0796198 & 432.14 & 552.14 \\
\hline 180 & 0.1294032 & 0.1255930 & 376.56 & 556.56 \\
\hline 240 & 0.2105297 & 0.1813350 & 319.80 & 559.80 \\
\hline 300 & 0.3781504 & 0.2576560 & 259.87 & 559.87 \\
\hline 360 & 1.1034473 & 0.411207 & 183.27 & 543.27 \\
\hline 420 & 2625 & 262.925 & 0.32 & 420.32 \\
\hline 480 & 2625 & 262.925 & 0.32 & 480.32 \\
\hline 10 & 0.0014965 & 0.0061339 & 533.91 & 543.91 \\
\hline 30 & 0.0044956 & 0.0184303 & 517.71 & 547.71 \\
\hline 60 & 0.0090329 & 0.0370562 & 493.40 & 553.40 \\
\hline 120 & 0.0184090 & 0.0757285 & 444.68 & 564.68 \\
\hline 180 & 0.0285258 & 0.1179280 & 395.75 & 575.75 \\
\hline 240 & 0.0399087 & 0.1662790 & 346.53 & 586.53 \\
\hline 300 & 0.0533355 & 0.2248720 & 296.93 & 596.93 \\
\hline 360 & 0.0700851 & 0.3009020 & 246.94 & 606.94 \\
\hline 420 & 0.0925016 & 0.4088130 & 196.54 & 616.54 \\
\hline 480 & 0.1255007 & 0.5831950 & 145.76 & 625.76 \\
\hline
\end{tabular}
and $V_{1}=0.015 \mathrm{~mL}$ (lower part). The definitions of $t_{\mathrm{i}}, V_{\mathrm{P}, \text { stock, }} V_{\mathrm{Q}, \text { stock }}$ and $V_{1}$ may be found in the text

function of $t_{\mathrm{i}}$ displays some interesting characteristics. Usually, independently of the composition of $\mathrm{P}$ and $\mathrm{Q}$ in the dead volume, $t_{\mathrm{L}}$ increases with an increase of $t_{\mathrm{i}}$ meaning that the longer the time spent in the inhomogeneous state, the longer the Landolt times that are expected to be observed. This trend is unchanged when the dead volume contains species $Q$ in a relatively large concentration (see the bottom part of Table 3), which is much higher than the $[\mathrm{Q}]_{0}$ value belonging to the initial concentration in the case of homogeneously mixing the reactants in the entire volume. If, however, the amount of $\mathrm{P}$ present initially in the dead volume is increased compared to the previous case (see the upper part of Table 3), the trend mentioned above is reversed and the calculated Landolt time $\left(t_{\mathrm{L}}\right)$ starts to decrease as $t_{\mathrm{i}}$ increases further. In other words, it means that if the system spends enough time in the dead volume at a suitable concentration range, it may advance further, and upon homogenization the Landolt time might become smaller and smaller. Furthermore, careful inspection of Table 3 also reveals that in some particular cases the Landolt time was found to be significantly shorter compared to the one measured at a completely uniform concentration distribution. Without any exception, these cases belong to those situations when the system spends enough time in the inhomogeneous 
state at a suitable concentration range of the reactants to complete the nonlinear autocatalytic reaction in the dead volume. Thus, when the system is homogenized, the concentration of the autocatalyst reaches a critical level to ignite the nonlinear reaction essentially within a second by spreading out via diffusion, enhanced by the mixing process in the entire volume. These numerical results clearly suggest that any inhomogeneity present locally in a reaction mixture actually starts to increase the Landolt time, but in the case of an autocatalytic reaction, after a certain period of time and depending on the actual composition of the dead volume, the reaction may proceed so that the Landolt time to be determined may also be shorter than compared to the case when the system was completely homogeneous over the whole period of time. To our understanding, it means that $\mu$ obtained from the fitting procedure may refer to the efficiency of the mixing process. If the most probable Landolt time $(\mu)$ is larger than the one determined at a completely uniform concentration distribution, it may indirectly refer to a situation where the system spends a relatively short period of time in the inhomogeneous state so as to not provide enough time for the autocatalytic reaction to be advanced in such a way to significantly decrease the measured Landolt time. However, if the most probable Landolt time $(\mu)$ is smaller, then, most likely, the efficiency of the mixing process is so weak that the system may spend enough time in the inhomogeneous state to complete the autocatalytic reaction in the dead volume and the autocatalyst formed there may then easily spread out in the entire volume by diffusion, enhanced by the mixing process. To support this argument, it is worthwhile to investigate the data presented in Table 4, where the effect of the increasing size of the dead volume is studied.

Table 4 shows the effect of increasing the size of the dead volume on the Landolt time. The trend is quite similar to that discussed above, i.e., it clearly depends on the composition of the dead volume. If a large excess of $\mathrm{Q}$ is present in the dead volume even for a relatively long period of time $(420 \mathrm{~s})$, the Landolt time increases significantly with an increase of the

Table 4 Calculated Landolt times at different dead volumes at $t_{\mathrm{i}}=420 \mathrm{~s}$. $V_{1}=0.55 V_{\text {dead }}$ (upper part of the table) and $V_{1}=0.1 V_{\text {dead }}$ (lower part of the table). The initial conditions are as follows: $V_{P, \text { stock }}=2.0 \mathrm{~mL}$ and $V_{Q, \text { stock }}=$ $1.0 \mathrm{~mL}$. The definitions of $t_{\mathrm{i}}, V_{\mathrm{P} \text {, stock, }}, V_{\mathrm{Q} \text {, stock }}$ and $V_{1}$ may be found in the text

\begin{tabular}{lllll}
\hline$V_{\text {dead }}(\mathrm{mL})$ & $R_{t_{\mathrm{i}} \text {, dead }}(\mu \mathrm{M})$ & $R_{t_{\mathrm{i}}, \text { bulk }}(\mu \mathrm{M})$ & $t_{\mathrm{h}}(\mathrm{s})$ & $t_{\mathrm{L}}(\mathrm{s})$ \\
\hline 0 & 0 & 0.0654406 & 542.01 & 542.01 \\
0.1 & 2.798086 & 0.0634664 & 113.64 & 533.64 \\
0.15 & 2.798086 & 0.0624644 & 109.80 & 529.80 \\
0.225 & 2.798086 & 0.0609422 & 104.44 & 524.44 \\
0.3 & 2.798086 & 0.0593962 & 99.5 & 519.5 \\
0.375 & 2.798086 & 0.0578257 & 94.94 & 514.94 \\
0.45 & 2.798086 & 0.0562297 & 90.71 & 510.71 \\
& & & & \\
0.1 & 0.346114 & 0.0566498 & 141.02 & 561.02 \\
0.15 & 0.346114 & 0.0527175 & 151.80 & 571.06 \\
0.225 & 0.346114 & 0.0472947 & 166.75 & 586.75 \\
0.3 & 0.346114 & 0.0423522 & 183.11 & 603.11 \\
0.375 & 0.346114 & 0.0378039 & 200.05 & 620.05 \\
0.45 & 0.346114 & 0.0335759 & 217.48 & 637.48
\end{tabular}

dead volume. In an opposite case when both reagents (P and Q) are present in the dead volume at a suitable concentration range, this time is enough to ignite the autocatalytic reaction, therefore the calculated Landolt time becomes smaller and smaller when the size of the dead volume increases. It seems to be quite obvious because the amount of $\mathrm{R}$ formed increases with the increasing size of the dead volume, thus, when the system is homogenized, the elevated level of autocatalyst results in shorter Landolt times to complete the reaction in the entire volume. A straightforward consequence of these observations is that imperfect mixing does not necessarily accelerate the rate of a nonlinear reaction when relatively large volumes are used. It is just an opposite case to the one reported by Niedl et al. in the case of a microfluidic channel, ${ }^{27}$ where the imperfect mixing accelerated the rate of a nonlinear reaction.

From these results, it looks to be worthwhile to examine the effect of reagent composition on the calculated Landolt time. Table 5 displays the most important results of our calculations.

As can be seen in Table 5, the calculated Landolt time goes through a minimum as a function of the concentration of both species present in the dead volume and the minimum is always larger than the $t_{\mathrm{L}}$ value measured in the completely homogeneous state if $t_{\mathrm{i}}$ is small enough. Another interesting feature of these calculations is that under the given conditions, the system must remain in the inhomogeneous state for a relatively long period of time-even under continuous stirring conditions-to provide small $t_{\mathrm{L}}$ values. In our opinion, it provides a sound support to our hypothesis that indeed the initial inhomogeneity, the size and the lifetime of the dead volume determined by the mixing conditions explain the variation of the Landolt time measured for an individual kinetic run in the case of a highly nonlinear reaction when a statistically meaningful number of samples is obtained.

Table 5 Calculated Landolt times at different $V_{1}$ values at $t_{\mathrm{i}}=180 \mathrm{~s}$ and at $V_{\text {dead }}=0.375 \mathrm{~mL}$. The initial conditions are as follows: $V_{\mathrm{P}, \text { stock }}=2.0 \mathrm{~mL}$ and $V_{Q, \text { stock }}=1.0 \mathrm{~mL}$. The definitions of $t_{\mathrm{i}}, V_{\mathrm{P} \text {,stock }}, V_{\mathrm{Q} \text {, stock }}$ and $V_{1}$ may be found in the text

\begin{tabular}{lllll}
\hline$V_{1}(\mathrm{~mL})$ & $R_{t_{\mathrm{i}} \text {, dead }}(\mu \mathrm{M})$ & $R_{t_{\mathrm{i}} \text {, bulk }}(\mu \mathrm{M})$ & $t_{\mathrm{h}}(\mathrm{s})$ & $t_{\mathrm{L}}(\mathrm{s})$ \\
\hline 0.3 & 0 & 0.150667 & 369.62 & 549.62 \\
0.285 & 0.0270108 & 0.149044 & 367.47 & 547.47 \\
0.27 & 0.0515994 & 0.147365 & 365.45 & 545.72 \\
0.255 & 0.0740338 & 0.145633 & 364.32 & 544.32 \\
0.24 & 0.0944496 & 0.143846 & 363.26 & 543.26 \\
0.225 & 0.1128660 & 0.142007 & 362.51 & 542.51 \\
0.21 & 0.1291935 & 0.140116 & 362.09 & 542.09 \\
0.195 & 0.1432387 & 0.138174 & 362.03 & 542.03 \\
0.18 & 0.1547095 & 0.136182 & 362.37 & 542.37 \\
0.165 & 0.1632259 & 0.134141 & 363.14 & 543.14 \\
0.15 & 0.1683435 & 0.132052 & 364.43 & 544.43 \\
0.135 & 0.1695924 & 0.129916 & 366.29 & 546.29 \\
0.12 & 0.1665378 & 0.127733 & 368.79 & 548.79 \\
0.105 & 0.1588578 & 0.125504 & 372.00 & 552.00 \\
0.09 & 0.1464294 & 0.123230 & 375.96 & 555.96 \\
0.075 & 0.1294032 & 0.120911 & 380.66 & 560.66 \\
0.06 & 0.1082426 & 0.118548 & 386.06 & 566.06 \\
0.045 & 0.0837071 & 0.116021 & 392.22 & 572.22 \\
0.03 & 0.0567756 & 0.113692 & 398.62 & 578.62 \\
0.015 & 0.0285258 & 0.111200 & 405.51 & 585.51 \\
0 & 0 & 0.108666 & 412.61 & 592.61 \\
& & & & \\
& 0 & & &
\end{tabular}


At the same time, we are leaning toward expressing our doubt that local fluctuations in the later phase of a reaction in a completely homogeneous solution might play a decisive role to determine the stochasticity of this system.

As a next step, we interpret our experimental results in a stepwise fashion using the concept detailed above. First, we shall focus on the effect of the overall volume on the most probable Landolt time $(\mu)$. As demonstrated (see Fig. 2 and 3), $\mu$ displays a maximum as a function of the overall volume. This experimental observation may be rationalized if we consider that the dead volume is always present in practice in the case of an imperfectly stirred system. A dead volume may easily occur around the wall, on the surface of the solution, in the corners (if they exist ${ }^{11}$ ) and in the vortex created by the stirrer bar. Of course, the relative size of the dead volume compared to the overall volume increases when the reaction is carried out in a small overall volume. At the same time, the same stirrer bar with a constant rpm value much more effectively dissipates the reagent solutions upon initiation to provide such a condition that the autocatalytic reaction is completed in the dead volume within much less time than the Landolt time measured at a completely uniform concentration distribution (see the upper part of Table 4). If the overall volume is increased, then of course the relative size of the dead volume decreases, therefore the most probable Landolt time to be measured approaches the Landolt time belonging to the hypothetical completely homogeneous state. A further increase of the overall volume may even cause such a situation where the $\mu$ value becomes higher than the Landolt time of the homogeneous state. This case can be rationalized in terms of the concentration ratio of species $P$ and $\mathrm{Q}$ in the dead volume because an increase of the overall volume evidently increases the possibility of the appearance of extremities in $[\mathrm{P}]_{0 \text {,dead }}$ and $[\mathrm{Q}]_{0 \text {,dead }}$. However, when a critical overall volume is reached under the given conditions, then of course the solution present in the dead volume may survive for a longer period of time as a result of poor mixing conditions. It therefore creates a better chance to complete the autocatalytic reaction, thus $\mu$ starts to decrease again, as can be seen in Fig. 3 .

Second, we shall also explain why and how the place of initiation affects the most probable Landolt time. When the initiating agent is not injected into the middle of the vortex created by the stirrer bar, then this solution is distributed more evenly in the reactor. It is therefore expected that in the dead volume, the concentrations of species $\mathrm{P}$ and $\mathrm{Q}$ are closer to those values that may correspond to the hypothetically homogeneous state. Thus, according to Table 5, the measured Landolt times are expected to be lower. In the opposite case when the reaction is started by injecting the initiating solution at the center of the vortex, the mixing process is far from being efficient, thus creating a dead volume where one of the reagents is in an overwhelming excess compared to the other one. According to Table 5, the most probable Landolt time should increase, in complete agreement with our experimental findings.

Third, we shall focus on the results obtained using different stirring patterns of the stirrer bar. Of course, when the stirrer bar rotates around an unfixed axis-i.e. it wanders-it decreases the size of the dead volume as well as the time being spent by the poorly mixed solution in the dead volume. They both act to decrease the most probable Landolt time compared to the case when the stirrer bar rotates around a fixed axis, leaving part of the dead volume far from the stirrer bar essentially imperfectly mixed.

Fourth, we shall explain why and how the most probable Landolt time may be independent of the size of stirrer bar. A shorter stirrer bar creates a smaller vortex at the same stirring rate compared to the other case. Therefore, the size of the dead volume there decreases, thus it tends to decrease the most probable Landolt time. At the same time, around the wall of the reactor the dead volume may be well hidden from the mixing process, thus an extreme concentration ratio of the reactants may easily sit there for a long period of time. The latter case according to Table 5 acts reversely and it increases $\mu$. In the case of a longer stirrer bar, an opposite case is established: the size of the dead volume in the vortex increases and an extreme concentration ratio may easily be created there, meaning that the Landolt time tends to increase. At the same time, however, a longer stirrer bar better mixes the solution far from the vortex of the center, therefore the residence time being spent there by the solution decreases. Furthermore, the concentration ratio of the reactants established by the initiation cannot be so extreme compared to the previous case. Therefore this scenario tends to decrease the most probable Landolt time. These effects seem to compensate for each other, resulting in an apparent insensitivity of the size of stirrer bar under the given conditions. It, however, does not necessarily mean that this compensation effect can be measured in the case of other reactors because it should evidently depend on the stirring pattern, which is influenced greatly by the geometry of the reactor. This possibility may be further tested in the near future.

One open question still remains to be answered, namely: does this stochastic behavior in this system belong uniquely to the arsenous acid-iodate system or is the arsenous acid-periodate reaction alone capable of producing irreproducible kinetic curves? To unambiguously answer this question, the $\mathrm{pH}$ is set to nearly neutral conditions $(\mathrm{pH}=6.51)$ where the arsenous acid-iodate reaction is vanishingly slow. ${ }^{12}$ The reactor was a standard quartz cuvette with a $1 \mathrm{~cm}$ pathlength. The stirring process was carried out using a $0.9 \mathrm{~cm}$ long stirring bar. The composition of the initial reaction mixture was $0.38 \mathrm{mM}$ periodate and $0.72 \mathrm{mM}$ arsenous acid and the ionic strength was adjusted to $1.00 \mathrm{M}$ using sodium perchlorate. Five individual kinetic runs are visualized in Fig. 7. It unambiguously demonstrates that the periodate-arsenous acid reaction shows CCB by itself. This remarkable observation clearly suggests that the kinetics of the title reaction should further be studied in detail in the near future to establish a reliable kinetic model.

\section{Conclusion}

The result presented here is a natural continuation of our previous study performed on a different system where the 


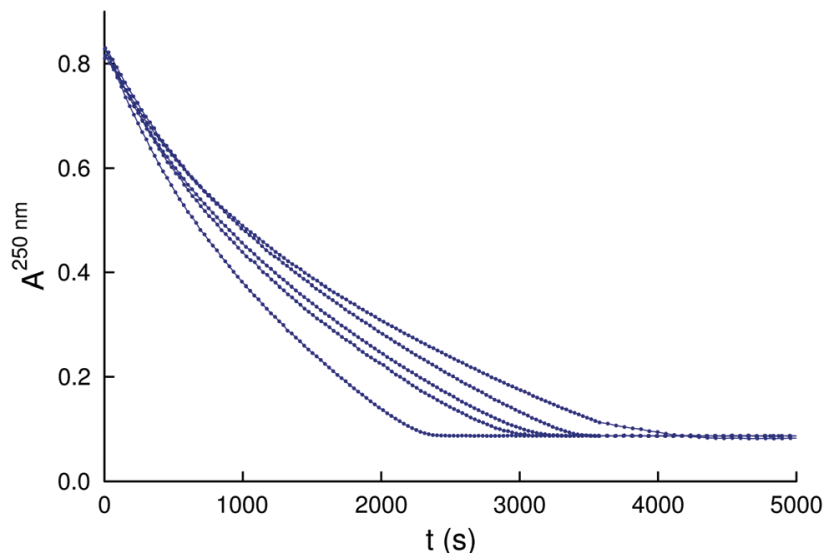

Fig. 7 Five individual kinetic runs in the periodate-arsenous acid system The absorbance was recorded using a spectrophotometer. The experimental conditions used are as follows: $\mathrm{pH}=6.51$ (carbonate buffer), $T_{\mathrm{H}_{x} \mathrm{IO}_{6}{ }^{(5-x)-}}^{0}=0.38 \mathrm{mM}, T_{\mathrm{H}_{3} \mathrm{AsO}_{3}}^{0}=0.72 \mathrm{mM}$, and $I=1 \mathrm{M}\left(\mathrm{NaClO}_{4}\right)$ in a standard $1 \times 1 \mathrm{~cm}$ cuvette with a $0.9 \mathrm{~cm}$ diameter cylindrical stirrer magnet.

origin of stochasticity is intended to be studied. ${ }^{11}$ Our preliminary result is confirmed here, that initial inhomogeneity indeed plays a significant role, but at the same time the present study unambiguously demonstrates that this is not the only decisive factor. We have clearly shown both experimentally and numerically that certain external physical factors characterizing the so-called stirring pattern also play a crucial role in determining the Landolt time belonging to the $50 \%$ probability. Among these factors, the size of the dead volume and the time being spent by the mixed reagents there have the greatest influence on the measured Landolt time in the case of an individual run. These major sources, of course, are mainly determined by the geometry of the reactor, stirring rate, place of initiation as well as the size and the rotation manner of the stirrer bar. We have also shown that these factors may also have a quite complex effect on the Landolt time measured. The most probable Landolt time can easily be prolonged compared to the one belonging to the completely homogeneous system, and shorter Landolt times can only be measured if the system spends enough time in the inhomogeneous state, within which the autocatalytic reaction may be ignited in the dead volume. Our calculations also revealed that it seems to be quite unlikely that local fluctuations appearing at a later phase of the reaction in the case of an initially homogeneous system play a decisive role.

Last but not least, it should also be noted that this study extended the number of real chemical redox systems featuring serious irreproducibility in the case of a statistically meaningful number of individual kinetic runs. Perhaps the most surprising result is that the arsenous acid-periodate reaction in itself is capable of exhibiting stochastic behavior, even if the arsenous acid-iodate reaction is completely uncoupled by an increase of $\mathrm{pH}$. The result is striking in a sense that in all of the known systems so far, the highly autocatalytic nature of the given reactions is experimentally justified. In this case however, the measured absorbance-time curves do not directly display the characteristic sigmoidal-shaped kinetic curve. Since a detailed and reliable kinetic model for the arsenous acid-periodate reaction has not yet been published in the literature, further experiments are being performed in our laboratory to unravel the secret of this fascinating system.

\section{Conflicts of interest}

There are no conflicts to declare.

\section{Acknowledgements}

The authors are grateful for the financial support of the Hungarian Research Fund NKFIH-OTKA Grant No. K116591. This work is supported by the European Union, co-financed by the European Social Fund Grant no.: EFOP-3.6.1.-16-2016-00004 entitled by Comprehensive Development for Implementing Smart Specialization Strategies at the University of Pécs, as well as by the GINOP-2.3.2-15-2016-00022 grant. The research was supported by the ÚNKP-17-3-I New National Excellence Program of the Ministry of Human Capacities. The present scientific contribution is dedicated to the 650th anniversary of the foundation of the University of Pécs, Hungary.

\section{References}

1 I. Nagypál and I. R. Epstein, J. Phys. Chem., 1986, 90, 6285-6292.

2 I. Nagypál and I. R. Epstein, J. Chem. Phys., 1988, 89, 6925-6928.

3 A. K. Horváth and I. Nagypál, Chem. Phys. Chem., 2015, 16, 588-594.

4 K. Soai, T. Shibata, H. Morioka and K. Choji, Nature, 1995, 378, 767-768.

5 K. Soai, I. Sato, T. Shibata, S. Komiya, M. Hayashi, Y. Matsueda, H. Imamura, T. Hayase, H. Morioka, H. Tabira, J. Yamamoto and Y. Kowata, Tetrahedron: Asymmetry, 2003, 14, 185-188.

6 P. Érdi and G. Lente, Stochastic Chemical Kinetics, SpringerVerlag, New York, 2014.

7 G. Lente, Comput. Chem. Eng., 2016, DOI: 10.1016/ j.compchemeng.2016.08.001.

8 T. Buhse, Tetrahedron: Asymmetry, 2003, 14, 1055-1061.

9 L. Xu, A. K. Horváth, Y. Hu, C. Ji, Y. Zhao and Q. Gao, J. Phys. Chem. A, 2011, 115, 1853-1860.

10 I. Lengyel, J. Li, K. Kustin and I. R. Epstein, J. Am. Chem. Soc., 1996, 118, 3708-3719.

11 L. Valkai, G. Csekö and A. K. Horváth, Phys. Chem. Chem. Phys., 2015, 17, 22187-22194.

12 G. Csekö, L. Valkai and A. K. Horváth, J. Phys. Chem. A, 2015, 119, 11053-11058.

13 L. Valkai and A. K. Horváth, Inorg. Chem., 2016, 55, 1595-1603.

14 S. Dushman, J. Phys. Chem., 1903, 8, 453-482.

15 J. R. Roebuck, J. Phys. Chem., 1904, 9, 727-763. 
16 M. C. Pagnacco, J. P. Maksimovic, N. I. Potkonjak, 22 E. Abel and A. Fürth, Z. Phys. Chem., 1923, 107, $313-328$. B. D. Bozic and A. K. Horváth, J. Phys. Chem. A, 2018, 122, 23 L. Valkai, G. Peintler and A. K. Horváth, Inorg. Chem., 2017, $482-491$.

56, 11417-11425.

17 T. S. Briggs and W. C. Rauscher, J. Chem. Educ., 1973, 24 G. Peintler, ChemMech, A Comprehensive Program Package for 50, 496.

18 R. M. Noyes and S. D. Furrow, J. Am. Chem. Soc., 1982, 104, $45-48$. Fitting Parameters of Chemical Reaction Mechanisms, 1989-2012, Version 2.1-5.0.

25 A. K. Horváth, J. Phys. Chem. A, 2007, 111, 890-896.

19 K.-R. Kim and D. J. Lee, J. Chem. Phys., 2002, 117, 2710.

20 D. R. Stanisavljev, M. C. Milenkovic, M. D. Mojovic and A. D. Popovic, J. Phys. Chem. A, 2011, 115, 2247-2249.

21 E. Abel and A. Fürth, Z. Phys. Chem., 1923, 107, 305-312.

26 A. K. Horváth, I. Nagypál and G. Csekö, J. Phys. Chem. A, 2008, 112, 7868-7872.

27 R. Niedl, I. Berenstein and C. Beta, Phys. Chem. Chem. Phys., 2016, 18, 6451-6457. 